\title{
Equilibrium spin currents and magnetoelectric effect in magnetic nanostructures
}

\author{
Patrick Bruno ${ }^{1}$ and Vitalii K. Dugaev ${ }^{2,3}$ \\ ${ }^{1}$ Max-Planck-Institut für Mikrostrukturphysik, Weinberg 2, 06120 Halle, Germany \\ ${ }^{2}$ Department of Physics and CFIF, Instituto Superior Técnico, Av. Rovisco Pais, 1049-001 Lisbon, Portugal \\ ${ }^{3}$ Frantsevich Institute for Problems of Materials Science, \\ National Academy of Sciences of Ukraine, Vilde 5, 58001 Chernovtsy, Ukraine
}

(Dated: 26 August 2005)

\begin{abstract}
We discuss the problem of equilibrium spin currents in ferromagnets with inhomogeneous magnetization. Using simple microscopic models we explain the physical origin of equilibrium spin currents. Next we derive the equilibrium spin current from the Hamiltonian with a gauge field associated with local rotations in the spin space. Several examples of magnetic systems are studied in details, and the persistent spin current is found to exist in the ground state of these systems. We also demonstrate the possibility to measure the equilibrium spin current using the magnetoelectrically induced electric field near the ring.
\end{abstract}

1. Introduction. The problem of generation of the pure spin currents attracted a lot of attention recently. It is mostly related to the perspectives of possible applications in spintronics, for which the generation and manipulation of spin currents is of a prime importance $\frac{1}{\underline{1}}$ Several different ways have been proposed to create the spin currents, like, for example, the injection of spin-polarized carriers from a magnetic metal or semiconductor, $\stackrel{2}{\rightleftharpoons}$ optical excitation of spin-polarized electrons or holes,$\frac{3}{,}$ equilibrium spin currents induced by the spiral states in ferromagnets,,$\frac{4}{4}$ spin Hall effect,,$\frac{5}{5}$ spin transport in presence of the spinorbit $(\mathrm{SO})$ interaction, $\underline{\underline{6}}$ and others.

Recently, the transport of magnetization by magnons has been studied by Meier et al $\underline{?}^{7}$ They demonstrated that by using a finite-length spin chain between magnetic reservoirs, the pure spin current can be generated without the transport of electron charge. Then Schütz et al proposed to use the magnons in a mesoscopic Heisenberg ring under an inhomogeneous magnetic field as a possible way to induce the persistent spin current. ${ }^{8}$ It was also found in these works. 7.8 that the magnetization transport by magnons is accompanied by an electric polarization, which can be experimentally observed in the vicinity of the magnetic wire ${ }^{7}$ or the mesoscopic ring. Most recently, Katsura et al used an electronic model of two transition atoms mediated by an oxygen atom, to show that the equilibrium spin current in this system is related to the electric polarization through a new mechanism of the magnetoelectric effect,,$\frac{9}{}$ which, in fact, is much stronger than the previously discussed ${ }^{7.8}$ relativistic mechanism.

At the same time, the theoretical studies of the magnetization transport provoked a lot of discussions about the existence and physical meaning of the non-vanishing equilibrium spin currents and about a proper definition of the spin current in the non-equilibrium transport phenomena ${ }^{10.11 .12}$ The main idea of these works was to re-define the transport spin current, which does not include the equilibrium (supercurrent) part responsible for the spin torque $\underline{\underline{10}}$

Here we show that several key points concerning the equilibrium spin currents and electric polarization dis- cussed in the above-mentioned papers ${ }^{7.8 .9 .12}$ should be further clarified. In particular, we emphasize that the equilibrium spin current (also called supercurrent because it is associated with the rigidity of the order parameter ${ }^{13}$ ) is related to a noncollinear magnetic ordering, and it exists at $T=0$ without magnons (in contrast to the claims of Refs. [8,12]). The details of electronic structure and/or SO interaction are not important for the equilibrium spin currents. On the other hand, the observable electric polarization is induced by the equilibrium spin current as well as by the magnon transport, and the corresponding mechanism necessarily includes the spin-orbit interaction. The magnitude of this effect strongly depends on the electronic structure of the material, so that it can be much larger than a bare relativistic effect ${ }^{9}$ We believe that the effective enhancement of the SO coupling for the magnetoelectric mechanism in the noncollinear magnets is an analogue of the renormalization (giant enhancement) of the Rashba SO interaction in the layered systems, as well as the reinforcement of the anomalous Hall effect, which is also related to the SO interaction.

2. Two spins in local fields. We start from a simple example demonstrating that the equilibrium spin current does not vanish for two interacting quantum spins $S=1 / 2$. Namely, we consider the Hamiltonian with interacting spins $S_{1}$ and $S_{2}$ in local magnetic fields $\mathbf{B}_{1}$ and $\mathbf{B}_{2}$

$$
H=-J \mathbf{S}_{1} \cdot \mathbf{S}_{2}-\mathbf{B}_{1} \cdot \mathbf{S}_{1}-\mathbf{B}_{2} \cdot \mathbf{S}_{2},
$$

where $\left|\mathbf{B}_{1}\right|=\left|\mathbf{B}_{2}\right|=B$. Calculating the time-derivative of spin operator $\dot{S}_{1 \mu}=\frac{i}{\hbar}\left[H, S_{1 \mu}\right]$ with (1), we find

$$
\dot{S}_{1 \mu}=\frac{J}{\hbar} \epsilon_{\mu \nu \lambda} S_{1 \nu} S_{2 \lambda}+\frac{1}{\hbar} \epsilon_{\mu \nu \lambda} S_{1 \nu} B_{1 \lambda} .
$$

The first term in the right side of (2) is the variation of spin related to the spin current $j_{1 \rightarrow 2}^{\mu}$, and the second one is related to the local field $\mathbf{B}_{1}$ acting on $\mathbf{S}_{1}$. In the equilibrium state, $\left\langle\dot{S}_{1 \mu}\right\rangle=0$ due to compensation of the moments transferred by the spin current and generated by the field $\mathbf{B}_{1}$. 
To calculate the value of spin current in the ground state, we use the perturbation theory assuming the interaction weak, $|J| / B \ll 1$. Let us take the quantization axis $z$ along the field $\mathbf{B}_{1}$, and the axis $y$ in the plane of vectors $\mathbf{B}_{1}$ and $\mathbf{B}_{2}$. In the limit of $|J| / B \ll 1$, the eigenfunction of Hamiltonian (1) corresponding to the ground state with energy $\varepsilon=-2 B$ is $\langle\psi|=\left\langle\psi_{1}\right| \otimes\left\langle\psi_{2}\right|$ with

$$
\left\langle\psi_{1}\right|=(1,0),\left\langle\psi_{2}\right|=\left(\cos \frac{\theta}{2},-i \sin \frac{\theta}{2}\right),
$$

where $\theta$ is the angle between the vectors $\mathbf{B}_{1}$ and $\mathbf{B}_{2}$.

Calculating the spin current in this state we find $\left\langle j_{1 \rightarrow 2}^{x}\right\rangle=-J \sin \theta / \hbar$, and $\left\langle j_{1 \rightarrow 2}^{y}\right\rangle=\left\langle j_{1 \rightarrow 2}^{z}\right\rangle=0$. The similar calculation gives us $\left\langle j_{2 \rightarrow 1}^{x}\right\rangle=-\left\langle j_{1 \rightarrow 2}^{x}\right\rangle$, which means nonconservation of the spin current in the presence of the local fields $\mathbf{B}_{1}$ and $\mathbf{B}_{2}$. The spin current transferred to the spin $\mathbf{S}_{1}$ generates the torque rotating this spin from the orientation along vector $\mathbf{B}_{1}$.

3. Hubbard model. This result can be explained by microscopic calculation using a two-site electronic model with Coulomb interaction. Let us consider a model of two-electron system with strong on-site Hubbard interaction $U$. This is an example of an electronic system, which is often used as a model leading to the localized magnetic moments with antiferromagnetic interaction. We show that by applying on-site magnetic fields $\mathbf{B}_{1}$ and $\mathbf{B}_{2}$ with different orientations, we induce the spin current transferring a spin moment. The Hamiltonian is

$$
\begin{aligned}
H=-t\left(c_{1 \alpha}^{\dagger} c_{2 \alpha}+c_{2 \alpha}^{\dagger} c_{1 \alpha}\right)+ & U \sum_{i=1,2}\left(c_{i \uparrow}^{\dagger} c_{i \uparrow} c_{i \downarrow}^{\dagger} c_{i \downarrow}\right. \\
& \left.-B_{i}^{\mu} c_{i \alpha}^{\dagger} \sigma_{\alpha \beta}^{\mu} c_{i \beta}\right),
\end{aligned}
$$

where $t$ is the hopping, $c_{i \alpha}^{\dagger}$ and $c_{i \alpha}$ are the creation and annihilation operators for electrons at the site $i$ with spin $\alpha$. We take $\left|\mathbf{B}_{1}\right|=\left|\mathbf{B}_{1}\right|=B$ and consider the case of strong on-site interaction and weak field, $B \ll t \ll U$.

The operator of the spin at site 1 is $\mathbf{S}_{1}=\frac{1}{2} c_{1 \alpha}^{\dagger} \boldsymbol{\sigma}_{\alpha \beta} c_{1 \beta}$, and the spin dynamics is determined by $\dot{\mathbf{S}}_{1}=i\left[H, \mathbf{S}_{1}\right]$, where the dot means time derivative. Using (4) we find

$\dot{S}_{1}^{\mu}=\frac{i t}{2}\left(c_{1 \alpha}^{\dagger} c_{2 \beta}-c_{2 \alpha}^{\dagger} c_{1 \beta}\right) \sigma_{\alpha \beta}^{\mu}+\epsilon^{\mu \nu \lambda} \sigma_{\alpha \beta}^{\nu} B_{1}^{\lambda} c_{1 \alpha}^{\dagger} c_{1 \beta}$.

Here the first term in the right hand site is the operator of spin current transferring spin from site 2 to site 1 . The second term is related to the force acting on the spin $\mathbf{S}_{1}$ by the the field $\mathbf{B}_{1}$. In the ground state $\left\langle\dot{\mathbf{S}}_{1}\right\rangle=0$, which can be satisfied due to the nonvanishing spin current

$$
j^{\mu}=\frac{i t}{2} \sigma_{\alpha \beta}^{\mu}\left\langle c_{1 \alpha}^{\dagger} c_{2 \beta}-c_{2 \alpha}^{\dagger} c_{1 \beta}\right\rangle_{0},
$$

flowing from site 2 to site 1 . The spin current transfers the angular moment compensating the force produced by the field $\mathbf{B}_{1}$.

We consider the system with two electrons, and take the basis of two-particle states $|\uparrow \downarrow, 0\rangle,|0, \uparrow \downarrow\rangle,|\uparrow, \uparrow\rangle,|\uparrow, \downarrow\rangle$, $|\downarrow, \uparrow\rangle,|\downarrow, \downarrow\rangle$. The Hamiltonian (4), and the operators of spin $\mathbf{S}_{i}$ and the spin current $\mathbf{j}$ can be presented as matrices in the basis of two-particle states. For example, if we take $\mathbf{B}_{1}$ along $z$, and $\mathbf{B}_{2}$ in the $y-z$ plane, the Hamiltonian in the new basis acquires the following form

$$
H=\left(\begin{array}{cccccc}
U & 0 & 0 & t & -t & 0 \\
0 & U & 0 & -t & t & 0 \\
0 & 0 & -B-B_{z}^{\prime} & i B_{y}^{\prime} & 0 & 0 \\
t & -t & -i B_{y}^{\prime} & -B+B_{z}^{\prime} & 0 & 0 \\
-t & t & 0 & 0 & B-B_{z}^{\prime} & i B_{y}^{\prime} \\
0 & 0 & 0 & 0 & -i B_{y}^{\prime} & B+B_{z}^{\prime}
\end{array}\right),
$$

where we denoted $B=B_{1 z}$ and $B_{\mu}^{\prime}=B_{2 \mu}$.

In the limit of strong interaction, $t / U \ll 1$, and $B=0$, this Hamiltonian can be transformed by a unitary transformation to the block form, in which the part describing the spin coupling is $\tilde{H}=J\left(\mathbf{S}_{1} \cdot \mathbf{S}_{2}-1 / 4\right)-\mathbf{B}_{1} \cdot \mathbf{S}_{1}-\mathbf{B}_{2} \cdot \mathbf{S}_{2}$, where $J=4 t^{2} / U$. By using the same transformation to the spin-current matrix, we find that it transforms into $\tilde{\mathbf{j}}=J \mathbf{S}_{1} \times \mathbf{S}_{2}$. This calculation shows that, microscopically, the equilibrium spin current results from the hopping of electrons between different sites, with an effective transfer of moment by the spin of electrons.

4. Noncollinear ferromagnet. Now we consider a textured ferromagnet, which represents a magnetic system in a topologically nontrivial metastable state. A simple example of such system is a magnetic ring with the easyaxis magnetic anisotropy, so that the magnetic moment, oriented along the ring, creates a vortex.

Let us take a continuous classical model of a ferromagnet described by the Hamiltonian, which includes the exchange interaction, anisotropy, and the interaction with an external magnetic field $\mathbf{B}(\mathbf{r})$ (this model was used recently to determine the Berry phase of magnons in textured ferromagnets 14 )

$$
H=\int d^{3} \mathbf{r}\left[\frac{a}{2}\left(\partial_{i} n_{\mu}\right)^{2}+\mathcal{F}\{\mathbf{n}(\mathbf{r})\}-\beta B_{\mu} n_{\mu}\right],
$$

where $\mathbf{n}(\mathbf{r})$ is the unit vector oriented along the magnetization at the point $\mathbf{r}, a$ is the constant of exchange interaction, $\mathcal{F}\{\mathbf{n}(\mathbf{r})\}$ is a function determining the anisotropy.

We will use the definition of spin current related to the transformation of the Hamiltonian under local rotations of vector $\mathbf{n}$ in the spin space $\frac{13}{13}$ This definition is in accordance with a general definition of currents in the quantum field theory $\frac{15}{15}$ In the case of a classical magnetic system with Hamiltonian (8), the corresponding transformations of the vector $\mathbf{n}(\mathbf{r})$ belong to the group $\mathrm{SO}(3)$. Thus, we perform a local rotation $\mathbf{n}(\mathbf{r}) \rightarrow \mathrm{R}(\mathbf{r}) \mathbf{n}(\mathbf{r})$ using the orthogonal transformation matrix $\mathrm{R}(\mathbf{r})=e^{i \psi(\mathbf{r}) J^{z}} e^{i \theta(\mathbf{r}) J^{y}} e^{i \phi(\mathbf{r}) J^{z}}$, where $\psi, \theta, \phi$ are the Euler angles determining the arbitrary rotations of the coordinate frame, and $J^{x}, J^{y}$, and $J^{z}$ are the generators of 3D rotations around $x, y$ and $z$ axes, respectively.

The Hamiltonian of exchange interaction (the first 
term in Eq. (8)) in the rotated frame has the form

$$
H_{e x}=\frac{a}{2} \int d^{3} \mathbf{r}\left(\partial_{i} n_{\mu}-A_{i \mu \nu} n_{\nu}\right)^{2},
$$

where the gauge field $A_{i}(\mathbf{r})=\left(\partial_{i} \mathrm{R}\right) \mathrm{R}^{-1}$. The matrix $A_{i}(\mathbf{r})$ can be presented as $A_{i}(\mathbf{r})=i J^{\mu} \mathcal{A}_{i}^{\mu}(\mathbf{r})$, where $\mathcal{A}_{i}^{\mu}(\mathbf{r})$ belongs to the adjoint representation of the group $\mathrm{SO}(3)$. Then the exchange energy can be written as

$$
H_{e x}=\frac{a}{2} \int d^{3} \mathbf{r}\left[\left(\partial_{i} \delta_{\alpha \beta}-i \mathcal{A}_{i}^{\mu} J_{\alpha \beta}^{\mu}\right) n_{\beta}\right]^{2},
$$

and the spin current density is defined as $j_{i}^{\mu}=$ $\gamma\left(\delta H / \delta \mathcal{A}_{i}^{\mu}\right)$, where $\gamma$ is the gyromagnetic ratio. We find

$$
j_{i}^{\mu}=-i c_{s} J_{\alpha \beta}^{\mu} n_{\beta}\left(\partial_{i} \delta_{\alpha \gamma}-i \mathcal{A}_{i}^{\delta} J_{\alpha \gamma}^{\delta}\right) n_{\gamma},
$$

where $c_{s}=\gamma a$. The spin current (11) is gauge invariant. We can fix the gauge by taking the auxiliary field $\mathcal{A}_{i}^{\mu}=0$. Then, using the relation $i J_{\nu \lambda}^{\mu}=\epsilon_{\mu \nu \lambda}$, we finally obtain

$$
\mathbf{j}_{i}=c_{s} \mathbf{n} \times \partial_{i} \mathbf{n}
$$

Thus, the spin current (12) is nonzero in the noncollinear ferromagnets. In particular, it is nonzero in a metastable state of the ferromagnet with topological excitations. 16 This definition is consistent with the spin-conservation equation relating the variation of the magnetization in time to the divergence of the spin current, ${ }^{8.17} n_{\mu}+$ $\operatorname{div} \mathbf{j}^{\mu}=T_{\mu}$, where $T_{\mu}=\gamma \epsilon_{\mu \nu \lambda} n_{\nu} B_{\lambda}^{e f f}$, and $\mathbf{B}^{e f f}$ is the local effective magnetic field, comprising the local external field and other local terms, such as magnetic anisotropies. The physical meaning is that of a hydrodynamic equation for $\mathbf{n}(\mathbf{r})$, where $T_{\mu}$ is a local source term (torque).

The thermodynamic average of the spin current is

$$
\left\langle j_{i}^{\mu}\right\rangle=c_{s} \epsilon_{\mu \nu \lambda}\left[\left\langle n_{\nu}\right\rangle \partial_{i}\left\langle n_{\lambda}\right\rangle+\left\langle\delta n_{\nu} \partial_{i} \delta n_{\lambda}\right\rangle\right]
$$

where $\langle X\rangle \equiv \operatorname{Tr}\left(X e^{-\beta H}\right) / \operatorname{Tr}\left(e^{-\beta H}\right)$, and $\delta X=X-\langle X\rangle$ is the fluctuation of $X$. Thus, there are two contributions to the spin current: the principal one arises from spatial variations of the average value of the magnetization axis, while the second one is due to the magnetization fluctuations (magnons). The magnon term is likely to be a small correction as compared to the principal (ground state) term. The ground state term can be interpreted as the supercurrent associated with the rigidity of the order parameter $\stackrel{4,13,18}{ }$ The magnon term has been discussed thoroughly, ${ }^{7.8 .19}$ and will not be discussed further here. In the following, we assume $T=0$, so that the magnon term vanishes (except for the effect of quantum zero-point fluctuations, which are usually negligibly small in ferromagnets). In this case, we can drop the angular brackets, which means that the thermodynamic average is implied.

5. Mesoscopic ring. To calculate the spin current in the ring geometry, it is convenient to use the cylindric coordinates $(\rho, \varphi, z)$ for the point on the ring. We assume that the vector $\mathbf{n}$ does not depend on $\rho$ and $z$. Then the exchange Hamiltonian $H_{e x}$ can be written as

$$
H_{e x}=\frac{a \zeta_{0}}{2 R} \int_{0}^{2 \pi} d \varphi\left(\partial_{\varphi} n_{\mu}+\frac{1}{2 \pi} \epsilon_{\mu \lambda \nu} \Phi_{\lambda} n_{\nu}\right)^{2}
$$

where $R$ and $\zeta_{0}$ are the radius and the cross section of the ring, respectively, $\Phi_{\lambda} \equiv L \mathcal{A}_{\varphi}^{\lambda}$, and $L=2 \pi R$.

Let us take $\mathcal{A}_{\varphi}^{\mu}$ constant along the ring. Then $\Phi_{\mu}$ is the flux of the $\mu$-component of the gauge field $\mathcal{A}_{\varphi}^{\mu}$ through the ring. Now we get the azimuthal spin current density in the ring as $j_{\varphi}^{\mu}=\left(\gamma / \zeta_{0}\right)\left(\partial H / \partial \Phi_{\mu}\right)$. Using (14) and taking $\Phi_{\mu}=0$, we find

$$
\begin{array}{r}
j_{\varphi}^{\rho}=\frac{c_{s}}{L} \int d \varphi\left[-n_{z}\left(n_{\rho}+\partial_{\varphi} n_{\varphi}\right)+n_{\varphi} \partial_{\varphi} n_{z}\right] \\
j_{\varphi}^{\varphi}=\frac{c_{s}}{L} \int d \varphi\left[n_{z}\left(-n_{\varphi}+\partial_{\varphi} n_{\rho}\right)-n_{\rho} \partial_{\varphi} n_{z}\right] \\
j_{\varphi}^{z}=\frac{c_{s}}{L} \int d \varphi\left[n_{\varphi}\left(n_{\varphi}-\partial_{\varphi} n_{\rho}\right)+n_{\rho}\left(n_{\rho}+\partial_{\varphi} n_{\varphi}\right)\right]
\end{array}
$$

In particular cases of the tangential magnetization $\left(n_{\varphi}=1, n_{\rho}=n_{z}=0\right)$, radial magnetization $\left(n_{\rho}=1\right.$, $n_{\varphi}=n_{z}=0$ ), and also for any intermediate case with the in-plane magnetization making a constant angle with the tangent vector, using (15)-(17) we obtain

$$
j_{\varphi}^{z}=2 \pi c_{s} / L, \quad j_{\varphi}^{\rho}=j_{\varphi}^{\varphi}=0 .
$$

It should be emphasized that the spin current (18) is related to the assumed metastable state of magnetization field but not to the magnons. The contribution of magnons exists for $T \neq 0$ but it is small because the magnons are weak excitations over the metastable state, completely vanishing in the limit of $T \rightarrow 0$.

The other example is a magnetic ring with uniaxial anisotropy in a homogeneous magnetic field along the axis $z$. Due to the anisotropy and exchange interaction, the magnetization along the ring is oriented with a certain angle out of the ring plane. There exist a metastable state a with crown-like magnetization profile in this system. (Due to the dipolar forces it can be the ground state.) We can calculate the angle $\theta$ using Hamiltonian (8) with $\mathcal{F}\{\mathbf{n}(\mathbf{r})\}=\lambda n_{z}^{2} / 2$ and $\lambda>0$, and the magnetic field $\mathbf{B}$ oriented along the axis $z$. Then, using the polar coordinates and assuming that $n_{\varphi}$ and $n_{z}$ do not depend on the coordinate along the ring, we find the energy of the metastable state

$$
E=\pi \zeta_{0} R\left[a n_{\varphi}^{2} /\left(2 R^{2}\right)+\lambda n_{z}^{2} / 2-\beta B n_{z}\right]
$$

and calculate the angle $\theta$ minimizing the energy (19) 14 $\cos \theta=\max \left\{1, \beta B /\left(\lambda-a / R^{2}\right)\right\}$ for $\lambda>a / R^{2}$, and $\theta=$ 0 for $\lambda \leq a / R^{2}$. The spin current in the ground state with $\theta \neq 0$ does not vanish. We calculate the components of it using Eqs. (15)-(17)

$$
j_{\varphi}^{\varphi}=\frac{2 \pi c_{s}}{L} \sin \theta \cos \theta, \quad j_{\varphi}^{z}=\frac{2 \pi c_{s}}{L} \sin ^{2} \theta, \quad j_{\varphi}^{\rho}=0 .
$$


Obviously, we obtain the same value of the spin current for different magnetization profiles if they can be transformed to each other by a global rotation.

6. Electric polarization. As discussed in Refs. 7 8], the spin current implies an electric polarization $P_{i}=$ $\epsilon_{i j \mu} j_{j}^{\mu} / c^{2}$, which is the relativistic effect of a transformation of magnetization to the electric field in the moving frame (this fact that has been known long $\operatorname{ago}^{20}$ ). The important point is that the electric polarization appears not only due to the spin transport of magnons but also due the spin supercurrent in the ground state (first term in Eq. (13)). This was shown by Katsura et al $\underline{\underline{9}}^{\frac{9}{n}}$ using a microscopic model with two transition atoms and an oxygen atom in-between. Their model calculations demonstrate that the magnetoelectric coefficient determining the magnitude of effect depends essentially on the material parameters. Taking it into account, we can write the polarization

$$
\mathbf{P}=\alpha_{m e}[\mathbf{n}(\nabla \cdot \mathbf{n})-(\mathbf{n} \cdot \nabla) \mathbf{n}],
$$

where we denoted by $\alpha_{m e}$ the magnetoelectric coefficient. This equation implies an enhancement of the bare relativistic effect in the condensed matter, i.e., it is due to the newly proposed magnetoelectric effect $\stackrel{9}{\underline{9}}$ It should be noted that the suitable materials for the observation of polarization are magnetic insulators and semiconductors because the induced electric polarization in a good metal would be completely screened by free electrons.

In the cases of the magnetic ring with the tangent magnetization along the ring or the constant radial magnetization, using (21) we find that the polarization vector $\mathbf{P}$ is oriented along the radius of the ring, and $P_{r}=-\alpha_{m e} / R$, where $R$ is the ring radius.

Let us estimate the magnitude of effect for a typical magnetic nanostructure, namely, a ferromagnetic disc of radius $R$ and thickness $h$ with a circular vortex domain (tangential magnetization) 21 The effect of the vortex can be approximated by replacing the disc by a ring of internal radius $r$ equal to the radius of the vortex core. In this case the polarization $\mathbf{P}$ is radial and decays as the inverse distance from the ring axis. We can approximately estimate the electrostatic potential $U$ at a point located on the axis at an altitude $z$ above the disc in the limit of $r, h \ll|z| \ll R$. We obtain $U \simeq-\alpha_{m e} h /|z|$. We estimate $\alpha_{m e}$ using the result of a three-atom model from Ref. 9] for the polarization per unit volume, which gives $\alpha_{m e} \simeq(e / a)(V / \Delta)^{3}$. Here $a$ is the lattice constant, $V$ is a hopping energy (hopping between the transition and oxygen atoms in [9]), and $\Delta$ is a characteristic electron energy (between $d$ and $p$ orbitals of different atoms). This formula was obtained for large SO splitting $\Delta_{S O} \gg \Delta$, and it does not explicitly depend the SO interaction. For a magnetic disc, using typical values $R=1 \mu \mathrm{m}, h=10 \mathrm{~nm}$, and $r=10 \mathrm{~nm}$, this yields $U \simeq 0.1(V / \Delta)^{3} \mathrm{~V}$ at an altitude $|z|=100 \mathrm{~nm}$. The vortex can be removed by application of an external magnetic field. The effect can be possibly detected experimentally by using a single-electron transistor as a detector of electric field.

We calculated the equilibrium spin current in mesoscopic systems, and showed that it does not vanish in the metastable state of magnetic system with topologically nontrivial magnetization profile. The electric polarization induced by the spin current can be experimentally measured.

This work is supported by FCT Grant POCI/FIS/58746/2004 (Portugal) and by Polish Grants PBZ/KBN/044/P03/2001 and 2 P03B 05325. V.D. thanks the Calouste Gulbenkian Foundation in Portugal for support.
1 G.A. Prinz, Science 282, 1660 (1998); S.A. Wolf et al., Science 294, 1488 (2001); I. Žutić, J. Fabian, S. Das Sarma, Rev. Mod. Phys. 76, 323 (2004).

2 Y. Ohno et al., Nature 402, 790 (1999).

3 S.D. Ganichev et al., Nature 417, 153 (2002).

4 J. König et al., Phys. Rev. Lett. 87, 187202 (2001).

5 J.E. Hirsch, Phys. Rev. Lett. 83, 1834 (1999); S. Murakami, N. Nagaosa, and S.C. Zhang, Science 301, 1348 (2003); J. Sinova et al., Phys. Rev. Lett. 92, 126603 (2004).

6 T.P. Pareek, Phys. Rev. Lett. 92, 076601 (2004).

7 F. Meier and D. Loss, Phys. Rev. Lett. 90, 167204 (2003).

8 F. Schütz, M. Kollar, and P. Kopietz, Phys. Rev. Lett. 91, 017205 (2003); Phys. Rev. B 69, 035313 (2004).

${ }^{9}$ H. Katsura, N. Nagaosa, and A.V. Balatsky, Phys. Rev. Lett. 95, 057205 (2005).

10 P. Zhang et al, cond-mat/0503305 (2005).

11 E.I. Rashba, Phys. Rev. B 68, 241315(R) (2003); 70, 161201(R) (2004); cond-mat/0408119 cond-mat/0507007

12 F. Schütz, P. Kopietz, and M. Kollar, Eur. Phys. J. B 41, 557 (2004).
13 P. Chandra, P. Coleman, and A. I. Larkin, J. Phys. Cond. Mat. 2, 7933 (1990).

14 P. Bruno, Phys. Rev. Lett. 93, 247202 (2004); Erratumibid. 94, 239903 (2005); V.K. Dugaev, P. Bruno, B. Canals, and C. Lacroix, Phys. Rev. B 72, 024456 (2005).

15 C. Itzykson and J.-B. Zuber, Quantum Field Theory (McGraw-Hill, New York, 1980).

16 V.L. Pokrovsky, M.V. Feigel'man, and A.M. Tsvelick, In: Spin Waves and Magnetic Excitations 2, edited by A. S. Borovik-Romanov and S. K. Sinha (Elsevier, Amsterdam, 1988), p. 67.

17 A.C. Durst and P.A. Lee, Phys. Rev. B 62, 1270 (2000).

18 G. Tatara and H. Kohno, Phys. Rev. B 67, 113316 (2003); G. Tatara and N. Garcia, Phys. Rev. Lett. 91, 076806 (2003).

19 B. Wang et al., Phys. Rev. B 69, 174403 (2004).

20 J. Frenkel, J. Physik 37, 243 (1926).

21 T. Shinjo et al., Science 289, 930 (2000); A. Wachowiak et al., Science 298, 577 (2004). 